\title{
POLYNOMIAL PROBLEMS OF THE CASAS-ALVERO TYPE
}

\section{S. YAKUBOVICH}

Abstract. We establish necessary and sufficient conditions for an arbitrary polynomial of degree $n$, especially with only real roots, to be trivial, i.e. to have the form $a(x-b)^{n}$. To do this, we derive new properties of polynomials and their roots. In particular, it concerns new bounds and genetic sum representations of the Abel-Goncharov interpolation polynomials. Moreover, we prove the Sz.-Nagy type identities, the Laguerre and Obreshkov-Chebotarev type inequalities for roots of polynomials and their derivatives. As applications these results are associated with the known problem, conjectured by Casas-Alvero in 2001, which says, that any complex univariate polynomial, having a common root with each of its non-constant derivative must be a power of a linear polynomial. We investigate particular cases of the problem, when the conjecture holds true or, possibly, is false.

Mathematics subject classification (2010): Primary 26C05, 12D10, 41A05; Secondary 13F20.

Keywords and phrases: Casas-Alvero conjecture, Abel-Goncharov polynomials, polynomial ring, Rolle theorem, Viéte formulas, Sz.-Nagy identities, Laguerre inequalities.

\section{REFERENCES}

[1] E. CAS AS-Alvero, Higher order polar germs, J. Algebra 240, 1 (2001), 326-337.

[2] J. Draisma And J. P. DE Jong, On the Casas-Alvero conjecture, Eur. Math.Soc. Newsl. 80 (2011), $29-33$.

[3] H.-C. Graf von Bothmer, O. Labs, J. Schicho and C. van de Woestijne, The Casas-Alvero conjecture for infinitely many degrees, J. Algebra 316, 1 (2007), 224-230.

[4] T. Posltra, Convex hulls and the Casas-Alvero conjecture for the complex plane, Rose-Hulman Undegrad. Math. J. 13, 1 (2012), 33-42.

[5] Q.I. Rahman And G. SchmeIsser, Analytic Theory of Polynomials, Clarendon Press, Oxford, 2002.

[6] M.A. Evgrafov, The Abel-Goncharov Interpolation Problem, Gosudarstv. Izdat. Tehn.-Teor. Lit., Moscow, 1954 (in Russian).

[7] N. Levinson, The Gontcharoff polynomials, Duke Math. J. 11 (1944), 729-733.

[8] N. Levinson, Corrections to "The Gontcharoff polynomials", Duke Math. J. 12 (1945), 335.

[9] V.L. GONCHAROV, Recherche sur les derivées successives de fonctions analytiques. I. Generalization de la serie d' Abel., Ann. École Norm. 47 (1930), 1-78 (in French).

[10] A. Aptekarev, V. Kaliaguine, J. VAn Iseghem, The genetic sum representation for the moments of a system of Stieltjes functions and its application, Constr. Approx. 16, 4 (2000), 487-524.

[11] I.I. IBRAGIUMOFF, Sur quelques systémes complets de fonctions analytiques, Izv. Akad. Nauk SSSR, S.M. (1939), 553-568 (in French).

[12] A.P. Prudnikov, Yu.A. Brychkov And O.I. Marichev, Integrals and Series: Elementary Functions, Gordon and Breach, New York, 1986. 